\title{
Influence of mesoporous silica on powder flow and electrostatic properties on short and long term
}

\author{
G. Lumay ${ }^{\mathrm{a}, *}$, S. Pillitteri ${ }^{\mathrm{a}}$, M. Marck ${ }^{\mathrm{a}}$, F. Monsuur ${ }^{\mathrm{b}}$, T. Pauly ${ }^{\mathrm{b}}$, Q. Ribeyre ${ }^{\mathrm{c}}$, F. Francqui ${ }^{\mathrm{c}}$, \\ N. Vandewalle ${ }^{a}$ \\ ${ }^{\text {a }}$ GRASP Laboratory, CESAM Research Unit, University of Liège, Belgium \\ ${ }^{\mathrm{b}}$ W. R. Grace \& Co, Worms, Germany \\ ${ }^{\mathrm{c}}$ GranuTools, Awans, Belgium
}

\section{A B S T R A C T}

We study the effect of three types of mesoporous silica (MPS) particles on the flow of three common excipients: microcrystalline cellulose, lactose and maize starch. While MPS are commonly considered as excipient and also as drug delivery carrier, the effects of MPS as flow aid additive and as powder stabilizer are investigated. MPS particles, called additive in the present study, are found to decrease powder cohesiveness, in particular for powders having higher water content and higher initial cohesiveness. According to both particle and pore size of MPS particles, the effect can be immediate (for small MPS particles having small pore size) or on the longer term (for larger MPS particles having higher pore size). Moreover, the electrostatic properties of the blends are modified by the presence of MPS. The quantity of electrostatic charge created in the blends during a flow in contact with stainless steel is decreased by the addition of MPS. We show that this decrease is induced by a modification of electric resistivity.

\section{Introduction}

Granular materials, fine powders and nanostructured powders are widely used in pharmaceutical industrial applications [1-4]. In particular, excipients are necessary in many dry powder formulations (dry powder inhalers, tablets, and capsules) [5,6]. Therefore, any additional information regarding their behavior and any recommendation about the use of additives could have huge consequences for the optimization of industrial processes or to avoid technical issues (caking, clogging, noncompliance or unconformity of the by-product), in particular in the framework of continuous manufacturing [7]. Understanding the behavior of powders and the interactions between the grains (commonly called particles in pharmaceutical sciences) is also crucial to perform drug modeling $[8,9]$.

Powder behavior is influenced by (i) steric repulsions, (ii) friction forces (iii) cohesive forces and (iv) interaction with the surrounding gas $[10,11]$. The steric repulsion is related to the grain geometry. Friction forces are influenced by both the surface state (rough or smooth surface) and the chemical nature of the grains. Cohesive forces may be induced by the presence of liquid bridges, by electrostatic charges, by van der Waals interactions or more rarely by magnetic dipole-dipole interactions. The predominance of one of these forces depends on both the environmental conditions and the physico-chemical properties of the grains.
When two materials are rubbed, electric charges are exchanged at the surfaces. This contact electrification is an old fundamental scientific subject. However, despite the numerous studies dedicated to this subject, the fundamental mechanisms behind the triboelectric effect are not fully understood in powders and granular materials [12]. The electric charges created by triboelectric effects lead to uncontrolled electric field, electrostatic forces between the grains and/or between the grains and the container. The difficulties to understand triboelectric processes in powders are related to the non-equilibrium character of the triboelectric exchange and to the variety of mechanisms behind this effect: impact, rolling and sliding (or rubbing). Moreover, the nature of contact inside a powder (normal forces, contact areas, ...) are difficult to quantify. Powder electrostatics is a very active subject of investigation [12-19] with many applications related to pharmaceutical industries [20-22].

Moisture is known to affect both static and dynamic behaviors of granular materials [23] and is also affecting the stability of pharmaceutical solid formulations [24]. However, the effect of moisture is far from obvious due to the interplay with electrostatic effects $[16,25,26]$. Indeed, moisture influences both surface grains conductivity and capillary bridges formation. For low relative air humidity, the electrical conductivity necessary for charge dissipation is reduced. For high relative air humidity, the electrical conductivity increases and liquid bridges may be formed at the contacts between the grains, resulting in

\footnotetext{
* Corresponding author.

E-mail address: geoffroy.lumay@uliege.be (G. Lumay).
} 


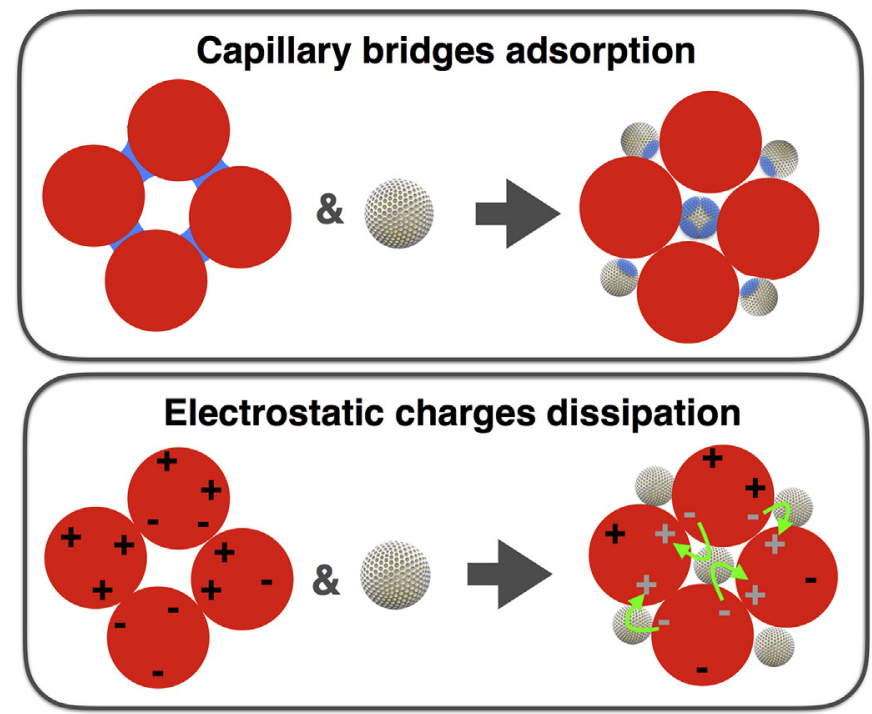

Fig. 1. Principle of the present study. MPS are playing a role on both capillary bridges and electrostatic charges to decrease cohesive forces.

sticking. Therefore, the electrical charges are dissipated more easily. However, the apparition of liquid bridges also induces cohesive forces inside the packing. At intermediate relative humidity values the cohesion is expected to be lower. Finally, it has been shown that air humidity modifies also van der Waals interactions [27].

Mesoporous silica (MPS) is interesting powders for pharmaceutical applications because of their adjustable porosity, high surface area, high density of OH-groups, inertness and good biocompatibility [28-32]. They are used as glidant, carriers, moisture stabilizer and to ensure stability of an amorphous state. MPS is also considered as an excipient improving tableting processes. Moreover, effect on powder electrostatics is expected [17]. Compared to fumed silica used classically as flow aid additive in powder industry, MPS is less pulverulent and has a higher moisture adsorption capacity. Finally, MPS are considered as drug delivery carrier in some applications.

In the present paper, we show how a small fraction of mesoporous silica (MPS) particles modifies the flowing properties of three common excipients. Therefore, MPS particles are considered as flow aid additive. In particular, we show that these additives are playing a role on both capillary bridges and electrostatic charges (see Fig. 1) to decrease cohesiveness. For that, the rheological properties of the excipients with different types of MPS particles and with different concentrations are measured with GranuDrum instrument. In parallel, the electrostatic behavior of the blends is analyzed using both triboelectric and resistivity measurements. Moreover, the cohesiveness temporal evolution after the addition of MPS is analyzed. Beyond the particular case of excipient/MPS blends, the present paper shows how a flowability measurement method associated with a recently developed powder triboelectrometer can help understand the physical properties of powder blends. The effect of other porous materials could be considered [33] in the future.

\section{Materials and methods}

\subsection{Materials}

\subsubsection{Mesoporous silica}

Three MPS produced by Grace Company under the name Syloid ${ }^{\circledR}$ silica have been selected. Table 1 shows the main characteristics of the selected MPS and Fig. 2 shows micrographs. All the selected MPS are micrometric. Syloid ${ }^{\circledR}$ 244FP silica has the smallest average particle size and an intermediate pose size (PS). Syloid ${ }^{\circledR}$ AL-1FP silica particle size is
Table 1

Summary of powder characteristics: water content $w \mathrm{t} \%$, granulometric indicators obtained with laser diffraction method (Median $d(v, 0.5)$ and extreme values $d(v, 0.9)$ and $d(v, 0.1)$, pore sizes (PS) and surface area (SA).

\begin{tabular}{|c|c|c|c|c|c|c|c|}
\hline & & wt $\%$ & $\mathrm{~d}(\mathrm{v}, 0.1)$ & $d(v, 0.5)$ & $\mathrm{d}(\mathrm{v}, 0.9)$ & PS & SA \\
\hline & & $\%$ & $\mu \mathrm{m}$ & $\mu \mathrm{m}$ & $\mu \mathrm{m}$ & $\mathrm{nm}$ & $\mathrm{m}^{2} / \mathrm{g}$ \\
\hline Maize Starch & Maize & 14.4 & 8.8 & 13.6 & 21 & - & \\
\hline Pharmatose $^{\oplus} 200 \mathrm{M}$ & Lactose & 5.5 & 13.8 & 52.6 & 136.3 & - & \\
\hline Avicel ${ }^{\circledR}$ PH101 & Avicel & 4.5 & 19.3 & 51.1 & 107.1 & - & \\
\hline Syloid ${ }^{\circledR}$ 244FP silica & S244 & 3.5 & 1.5 & 3 & 4.7 & 17 & 300 \\
\hline Syloid $^{\circledR}$ AL-1FP silica & SAL1 & 1 & 2.7 & 8 & 17.7 & 2,5 & 700 \\
\hline $\begin{array}{l}\text { Syloid }^{\circledast} \text { XDP3050 } \\
\text { silica }\end{array}$ & SXDP & 3.7 & 32.2 & 50 & 85.7 & 25 & 300 \\
\hline
\end{tabular}
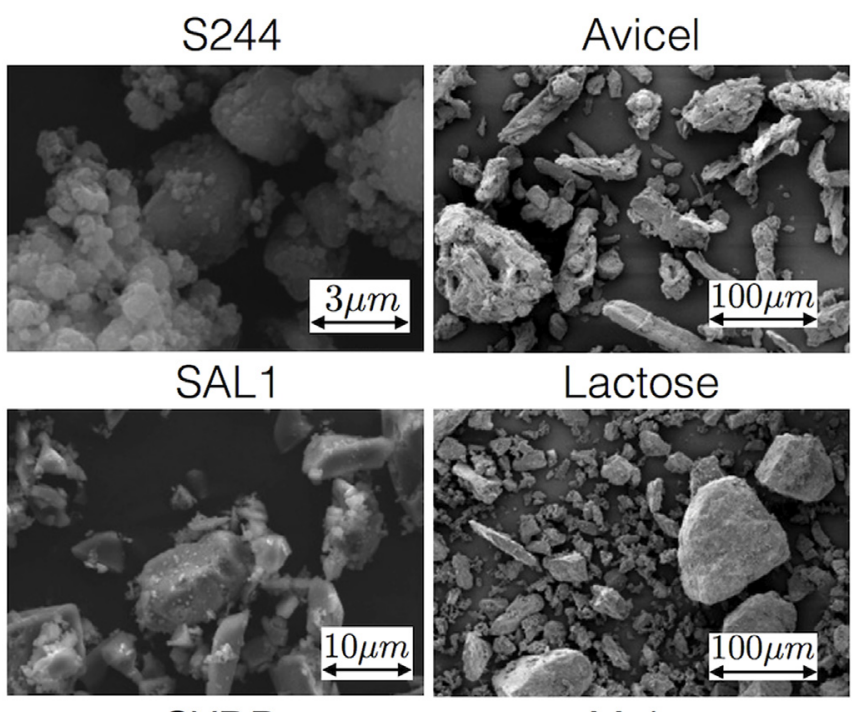

SXDP
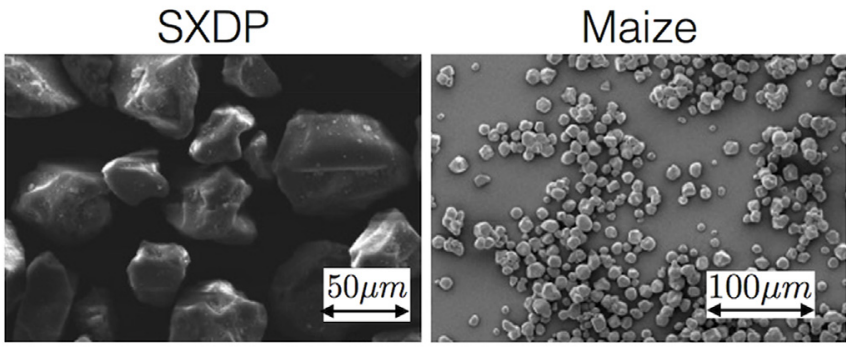

Fig. 2. Micrographs of MPS (right) and excipients (left).

slightly higher and has the lowest pore size. Finally, Syloid ${ }^{\circledR}$ XDP3050 silica has the highest particle size and also the highest pore size. All these MPS have low initial water content (see Table 1). In the present study, these MPS particles are considered as flow aid agent.

\subsubsection{Excipients}

Three excipients widely used in industry have been selected: Avicel ${ }^{\circledast}$ PH101, which is microcrystalline cellulose, Pharmatose ${ }^{\circledast} 200$ M, which is a mono-hydrate lactose and Maize starch. Microcrystalline cellulose and Lactose are crystalline whereas maize starch is semi-crystalline. Before the measurements presented in this paper, the excipients have been stored in our lab for at least one year after receiving them from collaborators. Therefore, the history of the samples is unknown and their properties could not correspond to the specifications of the producers for fresh powders. In addition to the water content wt $\%$, Table 1 shows the main granulometric indicators of the selected excipients and Fig. 2 shows micrographs. 


\subsection{Methods}

\subsubsection{GranuDrum}

The GranuDrum instrument is an automated powder flowability measurement technique based on the rotating drum principle. Powder and granular flow in this geometry has been widely studied from a fundamental point of view and the interest of the drum geometry for the measurement of the flowing properties of powders has been evidenced by different research teams [11,34-37]. A horizontal cylinder with vertical glass sidewalls called drum is half filled with the sample of powder. The drum rotates around its axis at an angular velocity ranging from 2 RPM to 10 RPM for the present study. This speed range has been selected to avoid the intermittent regime of low rotating speed and to be far from centrifugation. A CCD camera takes snapshots (50 images separated by $0.5 \mathrm{~s}$ ) at each angular velocity. The air/powder interface is detected on each snapshot with an edge detection algorithm. Afterward, the average interface position and the fluctuations around this average position are computed. Then, for each rotating speed, the flow angle (not considered in the present study) is computed from the average interface position and the dynamic cohesive index $\sigma$ is measured from the interface fluctuations. Indeed, interface fluctuations are induced by the cohesive forces between the grains. The dynamic cohesive index $\sigma$ is close to zero for non-cohesive powders and increases when the cohesive forces intensify. In addition, this method gives the opportunity to study complex rheological properties of powders (shear thinning, shear thickening and thixotropic behavior) by varying the rotating speed.

\subsubsection{GranuCharge}

GranuCharge is a recently developed triboelectric charger used to measure the total electrostatic charge created inside a granular material during a flow in contact with a selected material [26]. The sample is poured manually (the feeding could be automated) in a V-tube and flows to a Faraday cup. The V-tube is an assembly of two tubes of length $\mathrm{L}=350 \mathrm{~mm}$ and internal diameter $\mathrm{D}=47 \mathrm{~mm}$ to form a V shape with an angle of $90^{\circ}$. For the present study, $316 \mathrm{~L}$ stainless steel (S.S.) has been selected. The Faraday cup is connected to a dedicated electrometer able to measure electrostatic charges. At the end of the flow, the total value of the electric charge $\mathrm{Q}$ present in the powder is measured and the charge density $\mathrm{q}=\mathrm{Q} / \mathrm{m}$, where $\mathrm{m}$ is the sample mass, is computed.

\subsubsection{Resistivity}

The measurement cell described in the norm IEEE 548-1984 has been used to measure powder electrical resistivity $\rho_{\mathrm{e}}$. An electric potential difference $\Delta \mathrm{V}=500 \mathrm{~V}$ is applied by a power supply (Keithley 2260B-800-1) on a powder bed having height $\mathrm{h}=5 \mathrm{~mm}$ and the current $\mathrm{I}$ is measured with a pico ammeter (Keithley 6514). The measuring electrode surface is $S_{e}=507 \mathrm{~mm}^{2}$. The measurements were performed in ambient conditions at a temperature of $21{ }^{\circ} \mathrm{C}$ and a relative humidity of $35 \%$. The electrical resistivity $\rho_{\mathrm{e}}$ expressed in $\Omega . \mathrm{m}$ is computed with the relation

$\rho_{e}=\frac{\Delta V}{I} \frac{S_{e}}{h}$.

\subsubsection{Water content}

The water content of MPS were measured by Grace, while the excipients water content was measured with the following methods. A sample of mass $\mathrm{m}_{\mathrm{wet}}$ is spread in a crystallizing dish and placed in an oven at $110{ }^{\circ} \mathrm{C}$. The powder mass $\mathrm{m}_{\text {dry }}$ is measured after $24 \mathrm{~h}$. We checked after a second drying period of $24 \mathrm{~h}$ that the sample mass is stable. Finally, the water content $w \mathrm{t} \%$ is computed with the relation: wt $\%=100\left(\mathrm{~m}_{\mathrm{wet}}-\mathrm{m}_{\mathrm{dry}}\right) / \mathrm{m}_{\mathrm{dry}}$.

\subsubsection{Granulometry and microscopy}

Particle size distributions of excipients were measured in dry mode with a laser diffraction particle size analyzer (Malvern, Mastersizer 2000, Sirocco dispenser). The measurements are performed based on the MIE theory with the use of the general-purpose calculation model (normal sensitivity). The samples are analyzed in triplicate and the data presented correspond to the average value.

Micrographs were recorded with a scanning electron microscope (XL 30 FEG-ESEM, FEI) for MPS and (JEOL, JSM-IT300LV) for excipients.

\subsubsection{Mixing}

The mesoporous silica additives have been mixed with the excipients with a Y-shaped rotating mixer (Filtra, FTLMV-02) for $10 \mathrm{~min}$ at 38 rotations per minute. The volume of powder introduced in the mixer was $200 \mathrm{ml}$.

\subsubsection{Design of experiment}

Three excipients (Maize, Lactose and Avicel) were mixed with three fractions $(0.5 \%, 1 \%$ and $2 \%)$ of three types of MPS particles (S244, SAL1, SXDP). The excipients alone were also considered, leading to a set of 30 samples. Since water transfer between excipient and MPS particles was expected, we decided to perform the cohesiveness measurement after a repose time of at least $24 \mathrm{~h}$ following the mixing process. For a selection of five blends (Avicel $+2 \%$ S244, Lactose $+2 \%$ S 244 , Maize $+2 \%$ S 244 , Lactose $+2 \%$ SAL1 and Lactose $+2 \%$ SXDP) the evolution of the cohesiveness has been measured during a period of approximately two months. For all the samples, we stopped the measurements when the cohesiveness reached clearly a plateau. The electrostatic measurement has been performed at the end of this waiting period of two month to analyze stable blends. For the fastest dynamics, we repeated the mixing process and performed a measurement directly after the mixing to check the instantaneity of the effect of the MPS. Finally, in order to check the validity of our interpretation of the effect of MPS particles on the electrostatic charges, resistivity measurement has been performed.

\section{Results and discussion}

\subsection{Influence of MPS on the flow}

Fig. 3 shows the cohesive index $\sigma$ measured with GranuDrum as a function of the rotating speed for a selection of two excipients (Lactose and Maize starch) with $2 \%$ of S244 additive and also without any

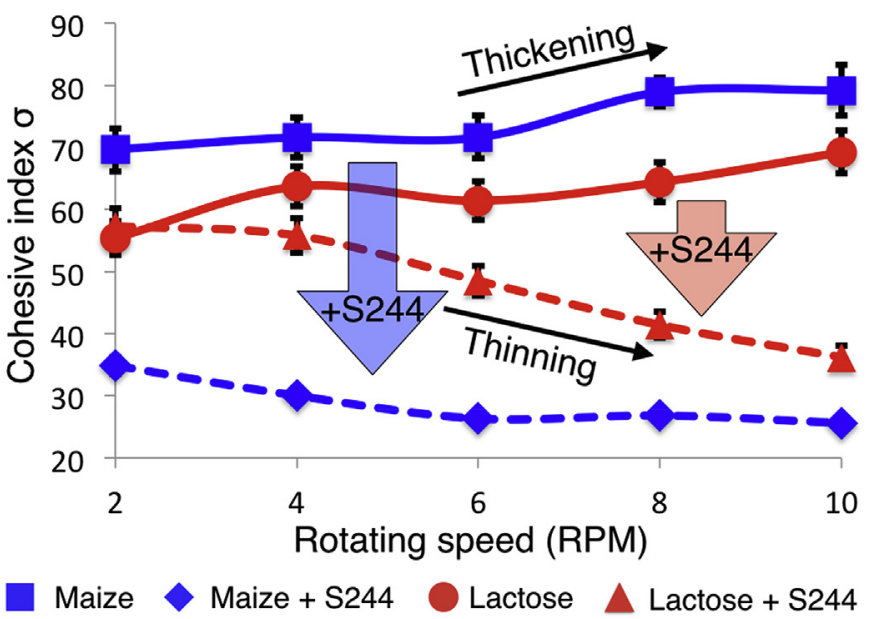

Fig. 3. Dynamic cohesive index $\sigma$ measured with GranuDrum as a function of the rotating speed for a selection of two excipients (Lactose and Maize starch) with and without $2 \%$ of S244FP additive. The error bars on Maize alone are corresponding to a standard deviation computed over five measurements. The other error bars are corresponding to a typical error of $5 \%$. 
additive. These two excipients have been selected to be plotted in Fig. 3 because Maize has the highest cohesiveness and because the effect of MPS on Lactose depends on the flow speed. The effect of the additive on maize starch cohesiveness is particularly important on the whole range of rotating speeds: the cohesiveness is drastically decreased. Moreover, the Maize starch associated with the additive shows a shear-thinning behavior (improvement of the flowability for high speeds) that could be useful for application needing high speed flows. Concerning Lactose, we do not observe any significant effect at low speed. However, due to shear-thinning behavior induced by the additive, the cohesiveness is lower for higher rotating speed. Therefore, in addition to the cohesiveness reduction, MPS particles are modifying the rheological properties. The measurement in GranuDrum with the excipients alone has been repeated five times to compute the standard deviation in order to estimate the measurement reproducibility. With the selected measurement parameters, the standard deviation is always lower than $5 \%$ of the average. In particular, the higher standard deviations were obtained with Maize (see error bars for Maize alone in Fig. 3) because the fluctuations are higher with highly cohesive powder. Therefore, this estimated error of $5 \%$ used to draw the error bars in the plots are corresponding to the worst-case scenario. Recording more images of the rotating drum can minimize this error.

In order to compare the effect of the whole set of additives on all excipients, we focus on the cohesiveness at a rotating speed of 10RPM. This rotating speed is moderate and corresponds to applications with typical flow speed of $45 \mathrm{~mm} / \mathrm{s}$. To estimate this flowing speed, we consider the linear speed of the drum wall that corresponds to the speed of the grains for non-cohesive granular materials [34] at the powder/air interface due to mass conservation. We would like to emphasize that this flowing speed evaluation is only intended to give a rough estimation and that the validity of this estimation will decreases when the powder cohesiveness will increases. Fig. 4 shows the cohesiveness at 10RPM for three MPS concentrations $(0.5 \%, 1 \%$ and $2 \%)$. The measurements were performed between 24 and $48 \mathrm{~h}$ after the mixing process. Globally, the effect of MPS on Avicel is low. Among the considered excipients, Avicel has the lowest cohesiveness and the lowest water content (see Table 1). Therefore, MPS additives do not have the opportunity to play a significant role on the flow. We will see hereafter that the additives are playing a role on electrostatic properties of Avicel. Concerning the other excipients (Lactose and Maize starch), both additives S244 and SAL1 are decreasing the cohesiveness. The effect of S244 is particularly important even at the lowest considered concentration (0.5\%). MPS efficiency on Maize and Lactose is certainly
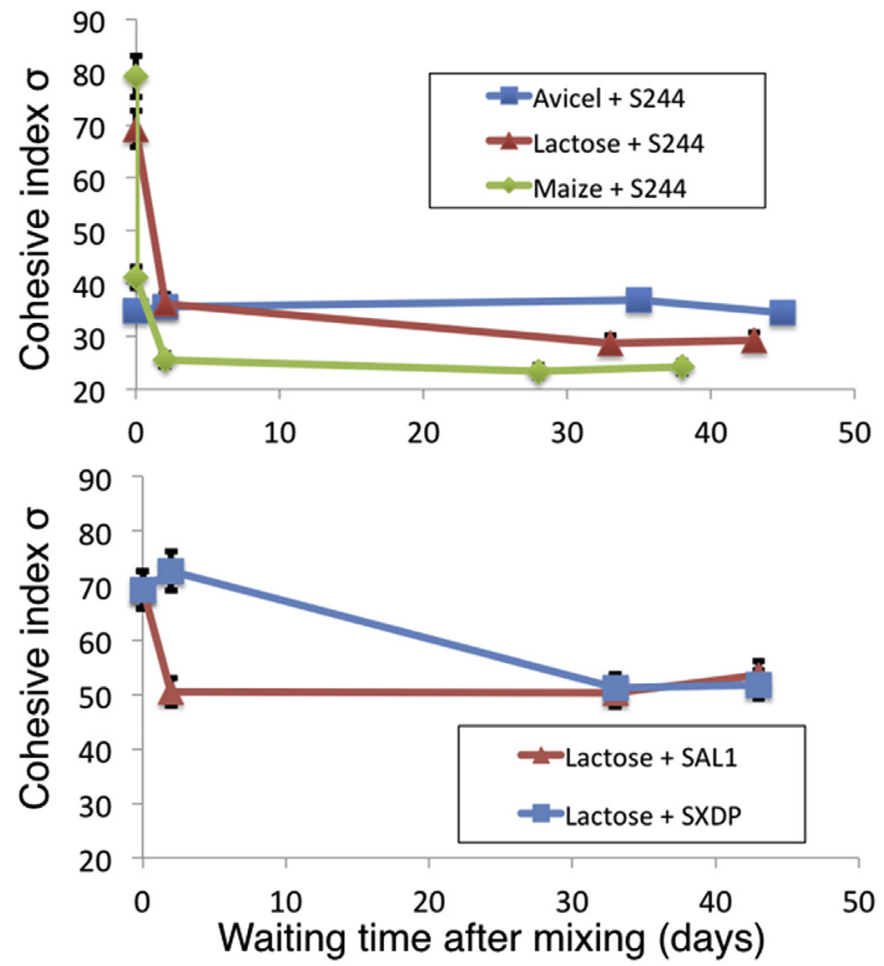

Fig. 5. Temporal evolution of powder blends cohesiveness at 10RPM. The error bars are corresponding to a typical error of $5 \%$.

due to their relatively high-water content (see Table 1). The small S244 particles are covering the larger excipients grains and are adsorbing efficiently the water at their surface. This scenario is depicted in Fig. 1. This water adsorption by MPS grains is expected to decrease capillary forces inside the bulk. On short term, the effect of SXDP corresponding to relatively larger grains with larger pore size is low. Therefore, on the short term, the cohesiveness decrease is more important with smaller MPS grains having small pore size and on excipients having higher water content and higher initial cohesiveness.

\subsection{Cohesiveness temporal evolution}

Since water exchange is expected to play an important role in the (a)
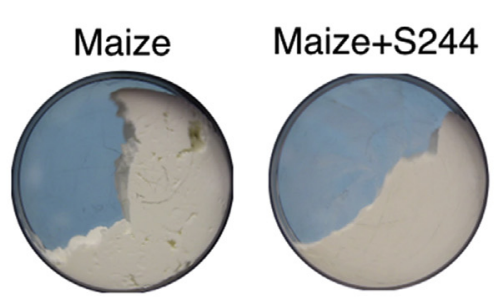

(b)
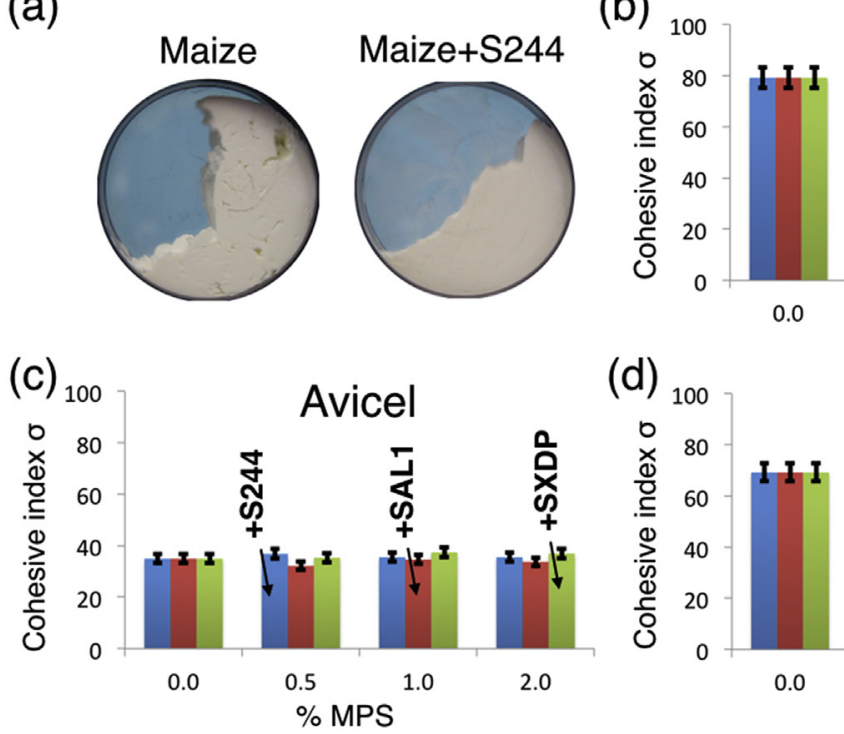

(d)

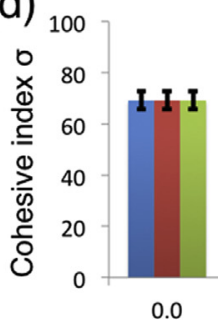

Maize

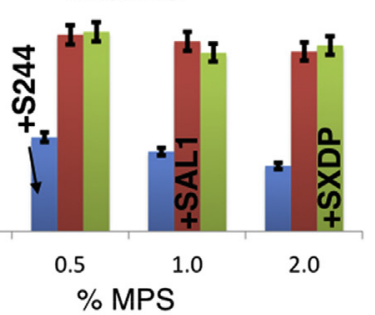

Lactose

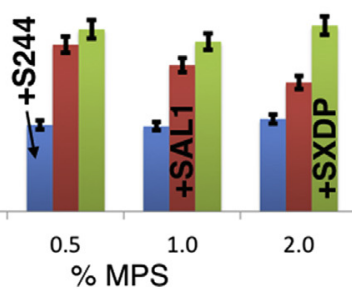

Fig. 4. (a) Typical pictures of the flow inside the rotating drum. (b-d) Variation of the dynamic cohesiveness $\sigma$ induced by the additives on the excipients at a rotating speed of 10RPM. The measurements were performed between one and two days after the mixing process. Blue, red and green bars are corresponding respectively to the addition of S244, SAL1 and SXDP MPS particles. Blue, red and green bars are corresponding respectively to the addition of S244, SAL1 and SXDP. The error bars are corresponding to a typical error of $5 \%$. (For interpretation of the references to colour in this figure legend, the reader is referred to the Web version of this article.) 
considered blends, a powder modification on the long term is possible. GranuDrum measurements have been repeated during a period of approximately two months with a selection of five blends. Fig. 5 (top) shows that the effect of S244 additive on the cohesiveness is immediate. Afterward, the cohesiveness is stable or decreases slightly to reach a plateau. Indeed, S244 MPS particles have small pore size and a higher surface area's containing a high density of $\mathrm{OH}$ groups. Therefore, the S244 MPS particles are able to bound more water to create a stable RH in the powder. This additive can be used to stabilize powder physical properties. The effect of SAL1 is lower but also immediate (see Fig. 5 (bottom)). This additive can also be used to protect moisture-sensitive ingredient. The larger SXDP particles having also the larger poresize do not modify cohesiveness on short term. However, a cohesiveness decrease is observed on the long term to reach the cohesiveness of the blend containing SAL1 additive. Therefore, these relatively big particles are able to load liquid on longer terms and can be used to incorporate liquids into powder formulations without degrading its flowability.

\subsection{Electrostatic charges}

It is well known that the effect of capillary bridges and electrostatic charges on powder cohesiveness is coupled $[16,25,26]$. Therefore, the electrostatic properties of the blends have been analyzed with the powder triboelectrometer called GranuCharge. In particular, the charge density obtained after a flow against $316 \mathrm{~L}$ stainless steel was measured for the excipients and for three concentrations of additive (see Fig. 6). The measurements where performed approximately 40 days after the mixing process with a relative air humidity of $32 \% \pm 2 \%$ at a temperature of $23{ }^{\circ} \mathrm{C} \pm 1{ }^{\circ} \mathrm{C}$. Therefore, the electrostatic measurements have been performed after stabilization of the cohesiveness. All the considered excipients charge negatively against stainless steel when they are alone. Taken as a whole, the addition of MPS decreases the ability of the blends to charge, even for low MPS concentrations. Even if the MPS do not modify significantly the cohesiveness of Avicel, the chargeability is found to decrease drastically. We will clarify this point in the section dedicated to electrical resistivity. Two blends are showing a more complex behavior: Lactose + S244 and Lactose + SAL1. Lactose powder alone charges negatively and the charge becomes slightly positive after the addition of MPS. During previous investigations, we observed that the sign of the charge density obtained with lactose could change with relative air humidity. In particular, positive charges were obtained for very low (less than $20 \%$ ) relative humidity. Since MPS are adsorbing the humidity at the surface of lactose grains, this transition from negative to positive electrostatic charges is not surprising.

\subsection{Resistivity}

The exchange of water between excipient grains and MPS modifies the water distribution inside the powder and thus a modification of electric resistivity is expected. Moreover, this resistivity modification will influence the tribo-electric properties of the powder. The resistivities of the excipients and of the blends with $2 \%$ of additives have been measured (see Fig. 7). Typically, powder resistivity measurements lead to significant fluctuations of the results because of the difficulty to generate a homogenous powder bed having always the same density in the cell. A repetition of the measurements for the excipients alone gives an estimation of $20 \%$ for the error. Concerning the excipients alone, Lactose and Avicel have almost the same resistivity while starch has a resistivity 1000 times lower. This could explain partially the lower chargeability of starch. With Avicel, the addition of MPS decreases drastically the resistivity and this decrease could explain the effect of MPS on electrostatic chargeability observed in the previous section. The effect of the additive on starch resistivity is weak because starch alone has already a relatively low resistivity, probably due to its high-water content. With all the considered excipients, the resistivity is decreased after the addition of MPS and the effect is particularly important with S244.

MPS grains are adsorbing the humidity contained in the excipients or at their surface. Therefore, the humidified MPS grains could produce a network having a low resistivity and this network could enhance the diffusion of electrostatic charge and then reduce the tribo-electric chargeability. This scenario is depicted in Fig. 1. In order to check that humidity decreases MPS resistivity, the resistivity has been measured with fresh MPS samples and with humidified MPS samples (see Fig. 7(a)). The humidified samples were simply in contact with ambient air for one week $\left(40 \% \mathrm{RH}\right.$ and $\left.21^{\circ} \mathrm{C}\right)$. The resistivity is found to decrease after this humidification process. In Fig. 7(a), the resistivity is plotted in logscale. Therefore, the resistivity decrease of MPS with humidity is important.

\section{Conclusion}

The effect of three types of MPS (different grain and pore sizes) on both flowability and electrostatic properties of excipients has been

(a)

(c)
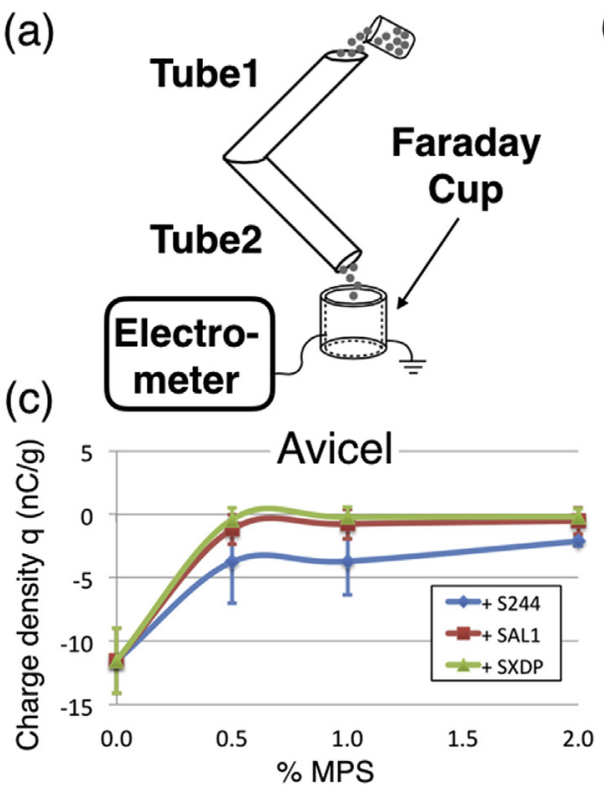

(b)

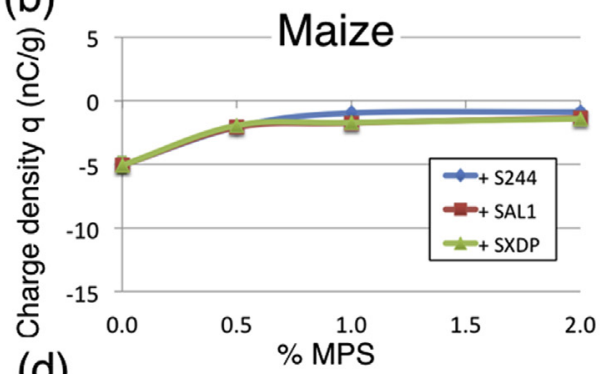

(d)

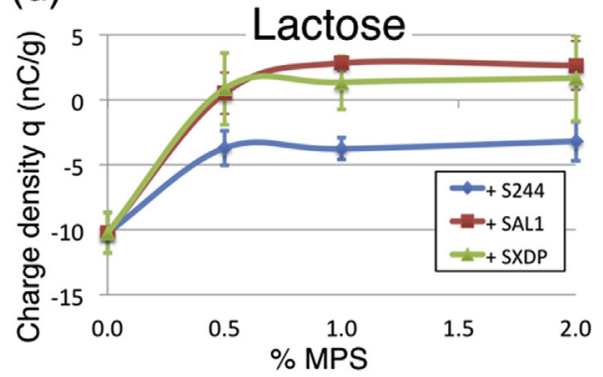

Fig. 6. (a) Sketch of GranuCharge instrument. (b-d) Charge density obtained after a flow against $316 \mathrm{~L}$ stainless steel for different MPS concentrations. In order to facilitate the comparison, the same charge density scale is used for the different plots. The error bars are corresponding to a standard deviation computed over three measurements. 

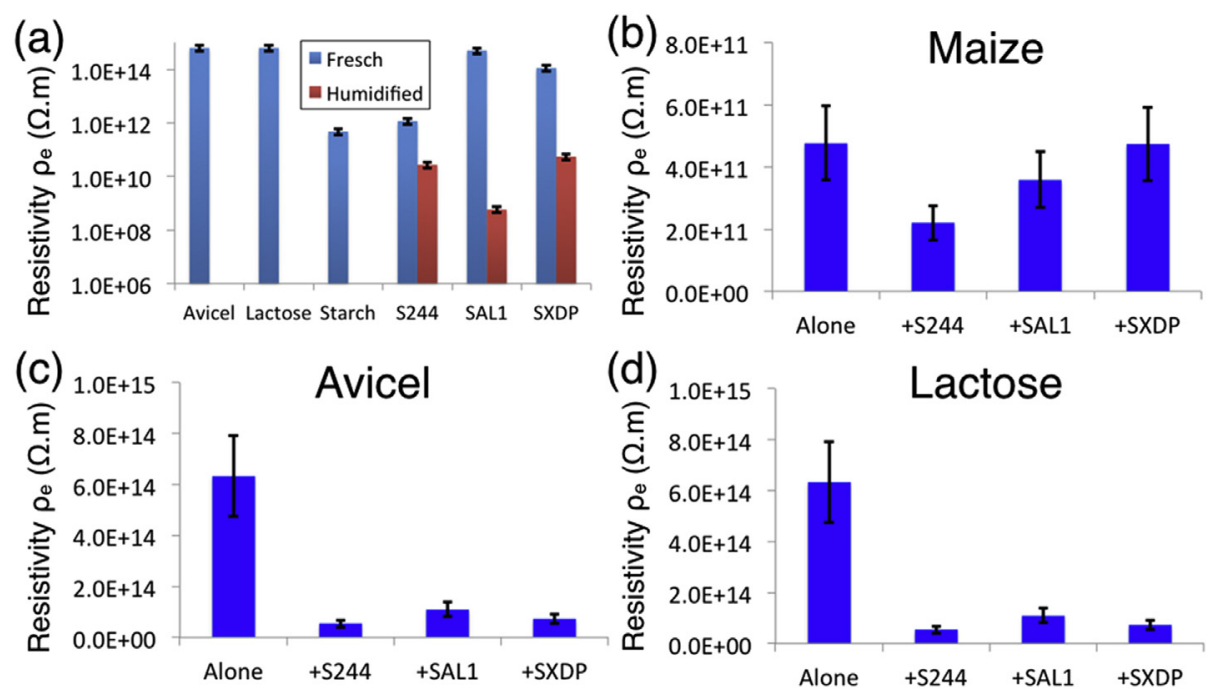

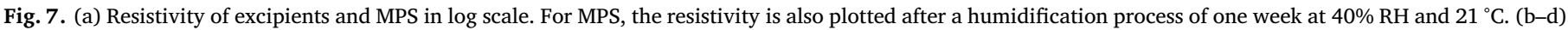
Resistivity of the excipients alone and of the blends with $2 \%$ of MPS. The error bars are corresponding to a typical error of $20 \%$.

analyzed. Taken as a whole, the excipient cohesiveness decreased after the addition of MPS. In addition to the cohesiveness reduction, MPS particles are modifying the powder rheological properties. In particular, a shear thinning behavior that could be interesting for high-speed processes is induced. The reduction of the cohesiveness is particularly pronounced for small MPS particles having small pore sizes (S244) associated with excipients having higher water content. With larger MPS grains (SXDP) having also a larger poresize, the cohesiveness decrease is observed only after a waiting time of a few days. Afterward, the cohesiveness still stable. The stronger and faster effect obtained with small MPS grains is probably due to the combination of a smaller poresize and a higher number of excipient/MPS contacts. Smaller pore size induces a faster capillary suction and MPS grains quickly adsorb the humidity contained at the surface of excipient grains.

The measurements performed with GranuCharge triboelectrometer show that the presence of MPS grains decreases the density of triboelectric charges produced during a flow. This effect could be explained by a decrease of the electric resistivity induced by MPS. The MPS grains are adsorbing the humidity contained in the excipients and are forming a network having a low resistivity, improving the diffusion of electrostatic charges. Therefore, MPS particles are playing a role on both capillary bridges and electrostatic charges to decrease cohesiveness.

A systematic study of the influence of both grain and pore size of MPS additive is a perspective. Moreover, the effects evidenced in the present paper with MPS could be investigated with other porous materials.

\section{Conflicts of interest}

The results presented in this paper were obtained in the framework of collaboration between researchers of the University of Liège, employees of the company GranuTools producing the measurement instruments GranuDrum and GranuCharge and employees of the company W. R. Grace \& Co producing the MPS. This collaboration was conducted without any financial transaction between the parties. W. R. Grace \& Co gives the samples for free.

\section{Acknowledgments}

This study is conducted in the framework of the "PowderReg" project, funded by the European programme Interreg VA GR within the priority axis 4 "Strengthen the competitiveness and the attractiveness of the Grande Région/Großregion". We thank our collaborators from UCB
Pharma (Luc Aerts and Frédéric De Smet) for the selection of the excipients and for fruitful discussions. Thanks to CAREM/ULiege for micrographs.

\section{References}

[1] F.J. Muzzio, T. Shinbrot, B.J. Glasser, Powder technology in the pharmaceutical industry: the need to catch up fast, Powder Technol. 124 (2002) 1-7.

[2] C.-Y. Wu, L. Dihoru, A.C.F. Cocks, The flow of powder into simple and stepped dies, Powder Technol. 134 (2003) 24-39.

[3] P. Ruenraroengsak, J.M. Cook, A.T. Florence, Nanosystem drug targeting: facing up to complex realities, J. Control. Release 141 (2010) 265.

[4] B. Van Snick, J. Dhondt, K. Pandelaere, J. Bertels, R. Mertens, D. Klingeleers, G. Di Pretoro, J.P. Remon, C. Vervaet, T. De Beer, V. Vanhoorne, A multivariate raw material property database to facilitate drug product development and enable insilico design of pharmaceutical dry powder processes, Int. J. Pharm. 549 (2018) 415-435.

[5] R.C. Rowe, P.J. Sheskey, M.E. Quinn, Handbook of Pharmaceutical Excipients, Pharmaceutical press, 2009.

[6] D.P. Eldera, M. Kuentz, R. Holmc, Pharmaceutical excipients - quality, regulatory and biopharmaceutical considerations, Eur. J. Pharm. Sci. 87 (2016) 88.

[7] N. Willecke, A. Szepes, M. Wunderlich, J.P. Remon, C. Vervaet, T. De Beer, Identifying overarching excipient properties towards an in-depth understanding of process and product performance for continuous twin-screw wet granulation, Int. J. Pharm. 522 (2017) 234-247.

[8] J. Siepmann, F. Siepmann, Mathematical modeling of drug delivery, Int. J. Pharm. 364 (2008) 328-343.

[9] W.R. Ketterhagen, M.T. am Ende, B.C. Hancock, Process modeling in the pharmaceutical industry using the discrete element method, J. Pharm. Sci. 98 (2009) $442-470$.

[10] P.G. de Gennes, Granular matter: a tentative view, Rev. Mod. Phys. 71 (1999) S374.

[11] G. Lumay, F. Boschini, K. Traina, S. Bontempi, J.-C. Remy, R. Cloots, N. Vandewalle, Measuring the flowing properties of powders and grains, Powder Technol. 224 (2012) 19-27.

[12] S. Matsusaka, H. Maruyama, T. Matsuyama, M. Ghadiri, Triboelectric charging of powders: a review, Chem. Eng. Sci. 65 (2010) 5781-5807.

[13] P.M. Ireland, Triboelectrification of particulate flows on surfaces: Part I Experiments, Powder Technol. 198 (2010) 189-198.

[14] P.M. Ireland, K. Nicholson, Analysis and comparison of particle tribochargers, Miner. Eng. 24 (2011) 914.

[15] S. Karner, N.A. Urbanetz, Arising of electrostatic charge in the mixing process and its influencing factors, Powder Technol. 226 (2012) 261-268.

[16] A. Schella, S. Herminghaus, M. Schroter, Influence of humidity on tribo-electric charging and segregation in shaken granular media, Soft Matter 13 (2017) 394-401.

[17] E. Šupuk, A. Zarrebini, J.P. Reddy, H. Hughes, M.M. Leane, M.J. Tobyn, P. Timmins, M. Ghadiri, Tribo-electrification of active pharmaceutical ingredients and excipients, Powder Technol. 217 (2012) 427-434

[18] S.R. Waitukaitis, V. Lee, J.M. Pierson, S.L. Forman, H.M. Jaeger, Size-dependent same-material tribocharging in insulating grains, Phys. Rev. Lett. 112 (2014) 218001.

[19] U. Zafar, F. Alfano, M. Ghadiri, Evaluation of a new dispersion technique for assessing triboelectric charging of powders, Int. J. Pharm. 543 (1-2) (2018) 151-159.

[20] J. Wong, P.C.L. Kwok, H.-K. Chan, Electrostatics in pharmaceutical solids, Chem. Eng. Sci. 125 (2015) 225-237. 
[21] D.A. Engers, M.N. Fricke, A.W. Newman, K.R. Morris, Triboelectric charging and dielectric properties of pharmaceutically relevant mixtures, J. Electrost. 65 (2007) 571-581.

[22] E.D. Lachiver, N. Abatzoglou, L. Cartilier, J.-S. Simard, Insights into the role of electrostatic forces on the behavior of dry pharmaceutical particulate systems, Pharm. Res. 23 (2006) 997-1007.

[23] N. Vandewalle, G. Lumay, F. Ludewig, J.E. Fiscina, How relative humidity affects random packing experiments, Phys. Rev. E 85 (2012) 031309.

[24] S. Airaksinen, M. Karjalainen, A. Shevchenko, S. Westermarck, E. Leppänen, J. Rantanen, J. Yliruusi, Role of water in the physical stability of solid dosage formulations, J. Pharm. Sci. 94 (2005) 2147-2165.

[25] G. Lumay, K. Traina, F. Boschini, V. Delaval, A. Rescaglio, R. Cloots, N. Vandewalle, Effect of relative air humidity on the flowability of lactose powders, J. Drug Deliv. Sci. Technol. 35 (2016) 207-212.

[26] A. Rescaglio, J. Schockmel, N. Vandewalle, G. Lumay, Combined effect of moisture and electrostatic charges on powder flow, EPJ Web Conf. 140 (2017) 13009.

[27] Y. Zhang, W. Pan, Describing van der Waals Interaction in diatomic molecules with generalized gradient approximations: the role of the exchange functional, J. Chem. Phys. 107 (1997) 7921.

[28] Y. Choudhari, H. Hoefer, C. Libanati, F. Monsuur, W. McCarthy, Mesoporous Silica Drug Delivery Systems, Advances in Delivery Science and Technology, Springer, New York, NY, 2014.

[29] R. Mellaerts, R. Mols, J.A.G. Jammaer, C.A. Aerts, P. Annaert, J. Van Humbeeck, G. Van den Mooter, P. Augustijns, J.A. Martens, Increasing the oral bioavailability of the poorly water soluble drug itraconazole with ordered mesoporous silica, Eur. J. Pharm. Biopharm. 69 (2008) 223-230.

[30] Y. Choudhari, U. Reddy, F. Monsuur, T. Pauly, H. Hoefer, W. McCarthy, Comparative evaluation of porous silica based carriers for lipids and liquid drug formulations, Open Mater. Sci. 1 (2014).

[31] H.A. Santos, J. Salonen, L.M. Bimbo, V.-P. Lehto, L. Peltonen, J. Hirvonen, Mesoporous materials as controlled drug delivery formulations, J. Drug Deliv. Sci. Technol. 21 (2011) 139-155.

[32] S. Saroj, S.J. Rajput, Composite smart mesoporous silica nanoparticles as promising therapeutic and diagnostic candidates: recent trends and applications, J. Drug Deliv. Sci. Technol. 44 (2018) 349-365.

[33] I. Jakob, M.A. Chairopoulou, M. Vučak, C. Posten, U. Teipel, Biogenic calcite particles from microalgae-Coccoliths as a potential raw material, Eng. Life Sci. 17 (2017) 605-612.

[34] J. Rajchenbach, Flow in powders: from discrete avalanches to continuous regime, Phys. Rev. Lett. 65 (1990) 2221.

[35] M.A.S. Quintanilla, J.M. Valverde, A. Castellanos, The transitional behavior of avalanches in cohesive granular materials, J. Stat. Mech. (2006) P07015.

[36] F. Boschini, V. Delaval, K. Traina, N. Vandewalle, G. Lumay, Linking flowability and granulometry of lactose powders, Int. J. Pharm. 494 (2015) 312-320.

[37] V. Rao Nalluri, M. Kuentz, Flowability characterisation of drug-excipient blends using a novel powder avalanching method, Eur. J. Pharm. Biopharm. 74 (2010) 388-396. 